\title{
Integrated Status Monitoring System for Ring Cabinet
}

\author{
Yang Sen ${ }^{1}$, Yao Zheng ${ }^{1}$, Gao Shan ${ }^{1}$, Li Lin ${ }^{1}$, Li Xueyan ${ }^{2}$, Zheng Xiaohu ${ }^{1}$, Zhou Jinghua ${ }^{3}$, Kang \\ Jun $^{3}$, Hu Yang ${ }^{3}$, Wang Qinghao ${ }^{3}$ Liu Xianfeng ${ }^{1}$, and Chen Fenghang ${ }^{1}$ \\ ${ }^{1}$ Dandong Power Supply Company, Liaoning Electric Power Company Limited, State Grid, China \\ ${ }^{2}$ Shibei Power Supply Company, Shanghai Electric Power Company Limited, State Grid, China \\ ${ }^{3}$ Fusun Power Supply Company, Liaoning Electric Power Company Limited, State Grid, China
}

Keywords: distribution network, ring cabinet, passive sensor, integrated state monitoring system

\begin{abstract}
In view of the frequent equipment failures caused by the heavy use of the distribution network ring cabinet, it is urgent to put forward the comprehensive state monitoring system of the distribution network ring cabinet. Timely find equipment insulation, condensation, local discharge, overheating and other defects. The system is equipped with real-time monitoring of the operating environment of the ring cabinet, the firefighting situation, the temperature of the heating parts, the working status of insulation parts, the intrusion behavior, etc., and the control of the temperature and humidity in the ring cabinet, the status information of the ring cabinet is transmitted to the rear monitoring platform, so as to facilitate the unified maintenance and management of the operation and maintenance personnel.
\end{abstract}

\section{Introduction}

In the urban distribution network, there are a variety of power supply modes of the distribution network, among which the power supply of the ring network cabinet has become the main form of the cable distribution network due to its reliability and economy. Therefore, the important electrical equipment in the ring network cabinet -the ring network cabinet has attracted more and more attention from users and state grid companies. At present, Dandong power supply company has a total of 1524 ring network cabinet, and the agricultural network ring network power supply cabinet equipment power supply equipment accounted for a growing proportion. As the total amount of installation of the distribution network ring cabinet is large, and most of them are located near communities and streets, the workload of operation and maintenance management is large. In addition, due to the poor operating conditions of the ring cabinet, structural limitations and procurement costs, PT, CT, lightning arresters, casings, pile heads and supporting insulators and other equipment are prone to abnormalities in long-term operation. After the occurrence of abnormal, if not handled in time, in the load changes, changes in the external environment (humidity, dust, etc.) or continued deterioration of abnormal, will cause equipment breakdown, explosion accident, etc. Thus, it can be seen that using modern communication technology and information technology to realize real-time online monitoring and status analysis of the ring cabinet can provide a basis for the state maintenance of the ring cabinet, greatly reducing the workload of operators and improving the power supply reliability of end users.

\section{The Cause of The Failure of The Ring Cabinet}

\subsection{Common Faults of Ring Cabinet}

\subsubsection{Failure of Secondary Control Circuit Breaking and Insulation Damage}

The failure of secondary control circuit breaking and insulation damage of $10 \mathrm{kV}$ loop cabinet are the main failures of the loop cabinet. The broken line of the secondary control loop of the circular network cabinet is caused by the improper contact of various wires and parts in the human or temporal parts. Line insulation damage is caused by aging insulation and over current and over 
voltage in the long-term operation of the cable of the ring cabinet. When the insulation problem occurs, the discharge is a continuous process, and causes the external insulation flashover of the bus-cabinet current transformer, and then causes the relative and interphase short circuit.

\subsubsection{Failure of Soft Connection and Cable Connector of Vacuum Circuit Breaker}

Network cabinet vacuum circuit breaker soft connection, cable joint fault is also prone to failure. In the soft connection of the circuit breaker of the ring network cabinet and the cable connector, the contact resistance is too large due to the joint oxidation and loose joint pressing, and the temperature gradually increases, which eventually leads to the soft connection of the bus and the cable connector burning out, or even the fire. Statistics show that $50 \%$ to $65 \%$ of cable accidents are caused by cable joints overheating, aging.

\subsubsection{Vacuum Circuit Breaker Fault}

Vacuum circuit breaker is the main break short circuit current of the ring network cabinet electrical equipment, its normal operation and performance related to the performance of the entire ring network cabinet vacuum circuit breaker there are several main faults: vacuum chamber leakage fault, rejection, mis operation fault, insulation fault, break and close fault and other faults.

\subsubsection{Ring Cabinet SF6 Gas Box Leakage Fault}

The leakage of SF6 gas box in the ring cabinet will lead to the decrease of SF6 gas pressure and the increase of water in SF6 gas box. Water and acid impurities together, will generate ammonia acid, corrosion of the gas box ring network cabinet; At low temperature, the water will condense on the surface of the solid insulation, and the flashover voltage along the surface will drop sharply, which is easy to cause insulation failure.SF6 air box leakage leads to the decline of insulation performance. The insulation performance of SF6 gas is closely related to the pressure of SF6.Only when the pressure of SF6 is not lower than a certain value can the insulation meet the requirements.

\subsection{Reasons for The Failure of Ring Cabinet}

\subsubsection{Working Environment}

Due to poor operating conditions, structural limitations and procurement costs, the equipment such as PT, CT, lightning arresters, casings, pile heads and supporting insulators are prone to abnormal insulation drop in long-term operation. After the insulation drops, if not handled in time, in the load changes, changes in the external environment (humidity, dust, etc.) or insulation continues to deteriorate, will cause equipment breakdown, explosion accident.

\subsubsection{Too High Temperature}

Ring network cabinet cable connector (t-head and elbow head) in operation due to abnormal contact, abnormal insulation or large load, will appear abnormal heating phenomenon, such as not timely treatment will lead to joint damage, grounding, short circuit and other accidents. In recent years, there have been many failures in the operation practice of ring network cabinet, such as cable joint heating, cable head explosion and ring network cabinet scrap. At the same time, due to the increase of electric load in recent years, the thermal fault of cable hair has a growing trend, and the cabinet can not be opened in operation, it is impossible to measure the actual temperature of the cabinet joints in operation, making it difficult to find and deal with the actual fault.

\subsubsection{Ambient Temperature}

Ring network cabinet is generally installed on the outdoor ground, located in the direct sunlight. In summer, the temperature in the ring network may exceed $60{ }^{\circ} \mathrm{C}$ due to the direct sunlight and heavy electric load. Winter temperature is low, the temperature can reach below zero; When it rains or the humidity is high, there will be condensation on the equipment surface; Heavy rain or urban waterlogging, the cable trench will be seriously flooded, in extreme cases, the ring cabinet also has the risk of flooding. At present, a lot of environmental control facilities (heater, dehumidifier and fan) are relatively simple, with high failure rate and no remote transmission function. The bad 
working environment of the ring cabinet is one of the main reasons for the insulation failure, the mechanical failure of the switch and the reduction of the service life of the equipment.

\section{Integrated Status Monitoring System for Ring Cabinet}

\subsection{System Introduction}

The comprehensive status monitoring system of the ring network cabinet adopts the hierarchical system structure. The front end is the status data acquisition layer, which contains all the data acquisition sensors arranged in the ring network cabinet, and is used to collect the information of the status of the ring network cabinet equipment, including the status monitoring sensor and the data acquisition terminal. The middle layer is the state concentration and processing layer, which is mainly the data collection concentrator arranged in the circular network cabinet. It is responsible for receiving and processing the data of the state monitoring and collection sensor, and uploading the data to the cloud platform, DTU equipment or the unified management platform of the power company. The back-end is the platform access layer, which is connected to the unified management cloud platform for data storage, processing, analysis and display, or connected to the unified management platform of power companies through DTU or secure and encrypted communication module. According to the function, the system is divided into five parts: temperature monitoring, environment monitoring, local release monitoring, intrusion monitoring and other monitoring.

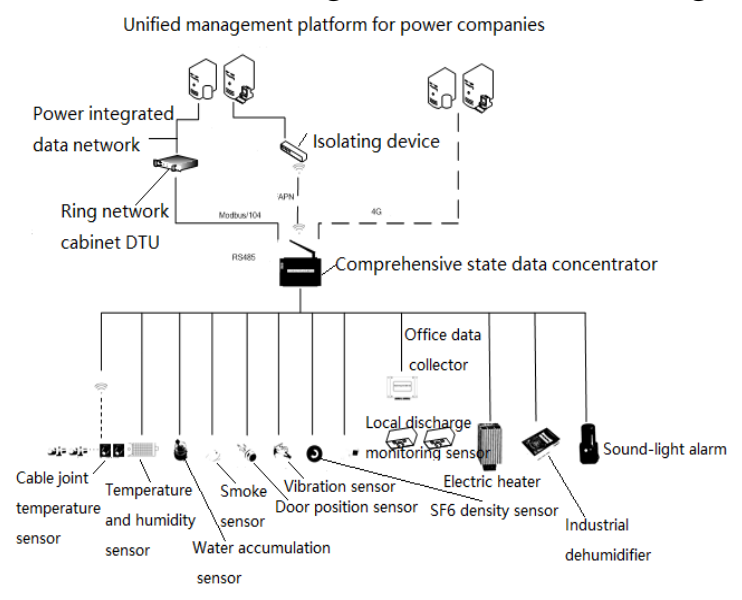

Figure 1 Architecture diagram of integrated state monitoring system of ring network cabinet

\subsection{Temperature Monitoring}

On-line monitoring unit for cable joint temperature. Cable joint temperature online monitoring the integrated use of advanced sensor technology, induction technology, low power wireless transmission technology, which can realize real-time temperature data collection and communication signal wireless transmission, historical data query, the temperature curve timely online exhibition, the limit temperature alarm, intelligent message warning functions such as, have a safe and practical, stable and reliable, economic operation, etc.

The on-line humidity monitoring unit of cable connector is composed of wireless temperature measuring terminal, data collector and remote monitoring platform. The temperature measuring terminal is arranged near the hair hot spot connected to the cable to obtain the temperature information and send the temperature signal to the data concentrator through Zigbee wireless communication. The data concentrator and the rear background can receive temperature information, analyze and process the temperature data, and alarm the temperature overshoot and phase temperature imbalance.

\subsection{Environmental Monitoring}

Monitor the temperature, humidity, cable water level and other information in the cabinet, and control the humidity and humidity in the cabinet, the equipment in the cabinet in the normal working range. Through the monitoring of environmental information, not only can understand the 
switch cabinet operation of the cabinet comprehensive environment, but also contact temperature, local insulation monitoring and alarm to provide reference data.

At present, there is generally no heater or dehumidifier installed in the circular network cabinet. Some of them are installed, but with low intelligence and high failure rate, so they cannot be monitored and controlled remotely. After the installation of this system can effectively monitor and control the environment of ring cabinet, greatly reduce the failure rate of ring cabinet equipment, improve the service life of equipment.

\subsection{Bureau Release Monitoring}

The on-line monitoring unit of partial discharge in the ring cabinet can monitor the discharge amplitude, frequency and discharge energy of the equipment in the ring cabinet in real time and analyze it. The partial discharge (pd) on-line monitoring unit of the ring cabinet can store the test spectrum and discharge trend, so as to timely discover the defects of the insulation equipment of the ring cabinet, and provide the criterion for evaluating its insulation level and aging degree, and provide the basis for the maintenance of the ring cabinet. This monitoring unit provides power frequency periodic discharge chart, two-dimensional spectrum chart, discharge trend chart, etc. The system adopts integrated anti-interference measures such as analog filtering, pulse grouping, periodic pulse elimination and dynamic threshold setting to make the test data real and reliable.

\subsection{Intrusion Monitoring}

The intrusion monitoring unit monitors whether the cabinet is normal, whether someone intrudes or destroys it, and if there is any abnormality, take photos and send them to the background.

\subsection{Other Monitoring}

In addition to the above monitoring functions, the system also has fire monitoring function, SF6 density monitoring function and DTU working power monitoring function. When fire action, SF6 density reduction and DTU power disconnection are detected, an alarm signal can be sent immediately through the data concentrator or the background, and an audible and visual alarm can be sent locally.

\section{Characteristics}

Ring network cabinet integrated online monitoring device can use advanced sensor technology, induction technology, low-power wireless transmission technology, which can realize real-time temperature data collection and communication signal wireless transmission, historical data query, the temperature curve timely online exhibition, the limit temperature alarm, intelligent message warning functions such as, have a safe and practical, stable and reliable, economic operation, etc.

Advanced technology, advanced design concept of equipment, can maintain its advanced through smooth upgrade, unified management of the distribution of different locations of the network cabinet integrated online monitoring concentrator, and provide analysis, processing and display functions.

High security, the solution from security, alarm and other aspects to take corresponding measures, the terminal side can be extended to use the distribution network security encryption communication chip, and in the provincial company deployed security encryption communication access platform.

High availability, practical, in the process of project design and implementation, always put the actual needs of safe and reliable operation of the network cabinet in the first place. Overall consideration of environmental conditions, monitoring objects, maintenance and other factors, the selection of practical equipment, so that the device is easy to operate, simple, efficient.

Easy maintenance, the system hardware can easily achieve remote management and maintenance, and provide a friendly man-machine interface, to ensure the easy maintenance of the system. 


\section{Conclusion}

As one of the most important equipment units for use and distribution, the number of ring network cabinets is very large. The integrated status monitoring system of ring network cabinet is suitable for monitoring the running status, intrusion and damage behavior of various types of ring network cabinet, and monitoring the running environment. The status information of the loop cabinet is transmitted to the rear monitoring platform. It realizes the integrity of the operation condition information in and out of the cabinet online monitoring, fundamentally reduces the operation and maintenance management, further solves the bad influence of information acquisition delay, and ensures the power supply reliability of end users.

\section{References}

[1] Zhuang Shining. Discussion on the design and implementation of status monitoring and fault warning system of ring network cabinet [J]. The world of communication. 21, 2015.

[2] Chen Jianping, Hu Zhanjiang, Su Xiaodong. Design of key technologies for on-line monitoring of mechanical characteristics of intelligent circuit breakers [J]. High voltage electrical appliances.04, 2014.

[3] Qu Hui, Li Haijun, Wu Libin, Zhang Haijun. The method and realization of the application system structured program design of single-chip microcomputer [J]. Journal of Inner Mongolia agricultural university (natural science). 01, 2010.

[4] Li Jianji. Development of ring network cabinet and load switch [J]. Jiangsu electric.11, 2008.

[5] Bai Tongyun. Electromagnetic compatibility layered and integrated design method -- a new method of electromagnetic compatibility design [J]. Electronic quality.05, 2008. 